\title{
A STUDY OF ROLE OF ANTIBIOTIC COATED BONE CEMENT IN MANAGEMENT OF INFECTED IMPLANT IN ORTHOPAEDIC SURGERIES
}

\author{
Raja Ashiq Ali Pulavan Ibrahim¹, Mathivanan Palaniappan², Kalyanasundaram Kolundan³, Srinivasan Anbu ${ }^{4}$
}

${ }^{1}$ Assistant Professor, Department of Orthopaedics, KAP Viswanatham Government Medical College, Tiruchirapalli.

2 Professor, Department of Orthopaedics, KAP Viswanatham Government Medical College, Tiruchirapalli.

${ }^{3}$ Associate Professor, Department of Orthopaedics, KAP Viswanatham Government Medical College, Tiruchirapalli.

${ }_{4}^{4}$ Associate Professor, Department of Orthopaedics, KAP Viswanatham Government Medical College, Tiruchirapalli.

\section{ABSTRACT}

\section{BACKGROUND}

Infection in Orthopaedic surgery is a disaster for both patient and the surgeon with an infected implant in a fracture surgery resulting in bad outcomes ranging from Osteomyelitis to non-union and septicaemia. In a majority of such cases, the infection settles only after removal of the implant. In our study, we tried to retain the implant by using antibiotic coated bone cement to get rid of the infection.

The aim was successful eradication of infection, with the patient remaining infection-free for 1 year.

\section{MATERIALS AND METHODS}

In our study, we chose 40 patients with infected implant with delayed union. All the patients were chosen carefully with fracture duration between 2 months to six months. Since the study material we chose was Vancomycin, we chose the patients who had MRSA infection. The bone involved was variable with fractures of Femur, Tibia and Humerus selected.

\section{RESULTS}

Forty patients were treated with antibiotic-coated bone cement beads for infected implant before union and 34 have achieved successful union, clinically free of signs of infection for more than 1 year. Six patients experienced a persistent infection necessitating removal.

\section{CONCLUSION}

Based on our experience, early aggressive debridement coupled with broad-spectrum antibiotic cement-coated beads are as effective as implant exit in control of infection and provides a better alternative. This approach permits fracture stabilisation and local delivery of high concentrations of broad-spectrum antibiotics and can reduce the number of debridement procedures required in the operating room.

\section{KEYWORDS}

Infected Non-Union, Antibiotic Loaded Cement, Antibiotic Elution, Vancomycin.

HOW TO CITE THIS ARTICLE: Ibrahim RAAP, Palaniappan M, Kolundan K, et al. A study of role of antibiotic coated bone cement in management of infected implant in orthopaedic surgeries. J. Evolution Med. Dent. Sci. 2017;6(7):581-585, D0I: $10.14260 /$ Jemds/2017/124

\section{BACKGROUND}

Infection in Orthopaedic surgery is a disaster for both patient and the surgeon with an infected implant in a fracture surgery resulting in bad outcomes ranging from Osteomyelitis to non-union and septicaemia. In a majority of such cases, the infection settles only after removal of the implant. In our study, we tried to retain the implant by using antibiotic coated bone cement to get rid of the infection. Antibiotic coated bone cement is a proven way to deliver high concentrations of the drug locally,(1) even to avascular areas that are inaccessible by systemic antibiotics.

The high concentrations of the drug achieved locally would be effective against organisms that are resistant to

Financial or Other, Competing Interest: None.

Submission 15-12-2016, Peer Review 11-01-2017,

Acceptance 17-01-2017, Published 23-01-2017.

Corresponding Author:

Dr. Raja Ashiq Ali Pulavan Ibrahim,

92/79 EB Colony,

Khajamalai,

Trichy-620023.

E-mail: ashiqalir@yahoo.com

DOI: $10.14260 /$ jemds $/ 2017 / 124$ drug concentrations achieved by systemic antibiotics. Also this delivery system provides only low serum antibiotic concentrations and hence less toxicity than that associated with systemic administration.

Over 30 years ago, Bulchoz and Engelbrecht reported that penicillin, erythromycin, gentamicin introduced into the cement used to stabilise the hip, spread into the surrounding tissues for months resulting in a prolonged local concentration of antibiotic.(2) After these findings, the interest in the application of cement impregnated with antibiotic in the treatment of Osteomyelitis grew. In 1979, as an alternative to the introduction of large deposits of antibiotic cement in the site of chronic Osteomyelitis, Klemm introduced gentamicin in cement beads and used them as temporary fillers for the gap that was created after the removal of necrotic tissue.(3) The cement impregnated with antibiotic (ALBC: Antibiotic Loaded Bone Cement), since the late 90 s, was increasingly used for the prevention of the Arthroplasty-related infections.(4,5) Also, over time, this choice has undergone significant changes and improvements concerning the chemical formulation, the techniques of preparation and clinical applications. 


\section{MATERIALS AND METHODS}

This is a prospective study of 40 patients satisfying the inclusion criteria conducted in the Department of Orthopaedics, Tirunelveli Medical College, during 2009 2015.

\section{Inclusion Criteria}

Infected implant before fracture union with a positive culture for MRSA, sensitive to Vancomycin. All the patients were chosen carefully with fracture duration between 2 months to six months. Since the study material we chose was Vancomycin, we chose the patients who had MRSA infection. The bone involved was variable, with fractures of Femur, Tibia and Humerus selected.

\section{Procedure}

As the first procedure, the fracture site was opened and a thorough saline wash was given. The area was then debrided thoroughly. The area was well prepared to hold the antibiotic beads. Then the antibiotic bead preparation begins.

\section{Preparation of Antibiotic Cement}

The polymethylmethacrylate monomer and powder must first be mixed together to form the liquid cement, and then the antibiotic is added. It is important to leave as many large crystals intact as possible to create a more porous mixture to increase the elution rate. Once the cement is formed, care should be taken when it is applied to bone.(6) Cement should be applied in the late stage of polymerisation to prevent interdigitation into bone while still allowing the surgeon some freedom to shape the articular surface of the bone. In our cases, we used a SS wire to string together the beads of antibiotics roughly $1 \mathrm{~cm}$ in diameter and the number of beads depended on the surface area of the region to be applied. The SS wires were fixed to the bone by drilling. The method used to mix the antibiotic seems to play an important role.(3) Hand mixing with a spatula has been shown to increase the total antibiotic release when compared with mixing with a specialised cement mixer. It is hypothesised that hand mixing introduces significant porosity to the cement, which in turn should increase antibiotic elution. Additionally, vacuum mixing of commercially available antibiotic-impregnated bone cement has been studied to determine its effect on antibiotic elution.

The PMMA enters the operating room packaged in monomer (Liquid) and polymer (powder) separately.(2) At the time of the preparation, when mixed, it becomes a viscous material paste, which solidifies in few minutes by an exothermic reaction. It acts as a fixation between the prosthetic components and the cancellous bone. During the mixing, pores of different sizes are produced as consequence of the chemical reaction and volume variations. These microholes may represent the start point of cracks and thus can be responsible for the premature failure of the cement. To avoid the formation of these pores, it is possible to prepare the cement under vacuum conditions. Nevertheless, the preparation under vacuum leads to a greater reduction in volume during the polymerisation, thus resulting in higher shrinkage and worse adhesion on the bone-prosthesis interface compared to the non-vacuum systems.

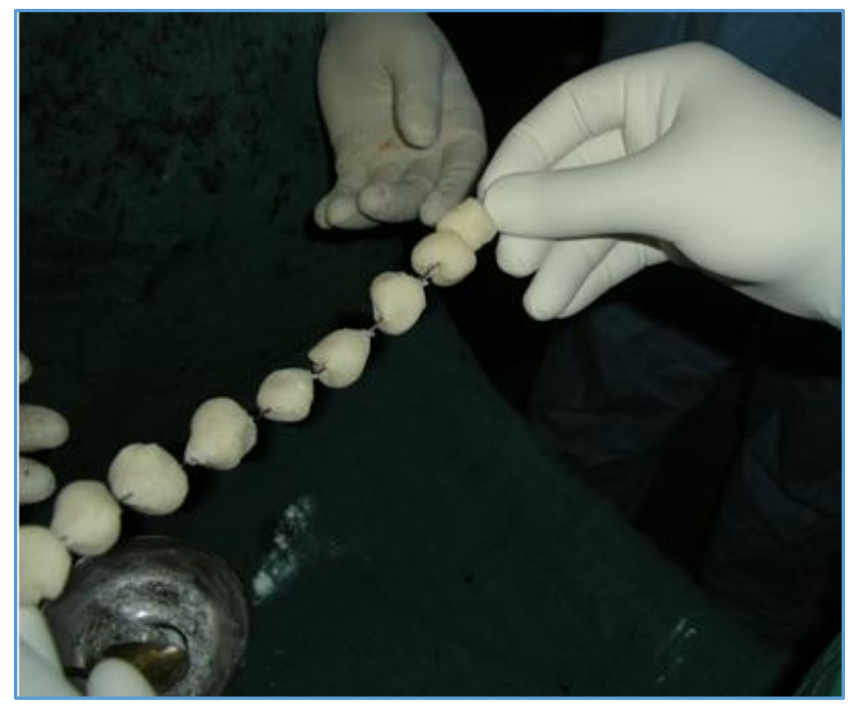

Figure 1. Preparation of Cement Beads

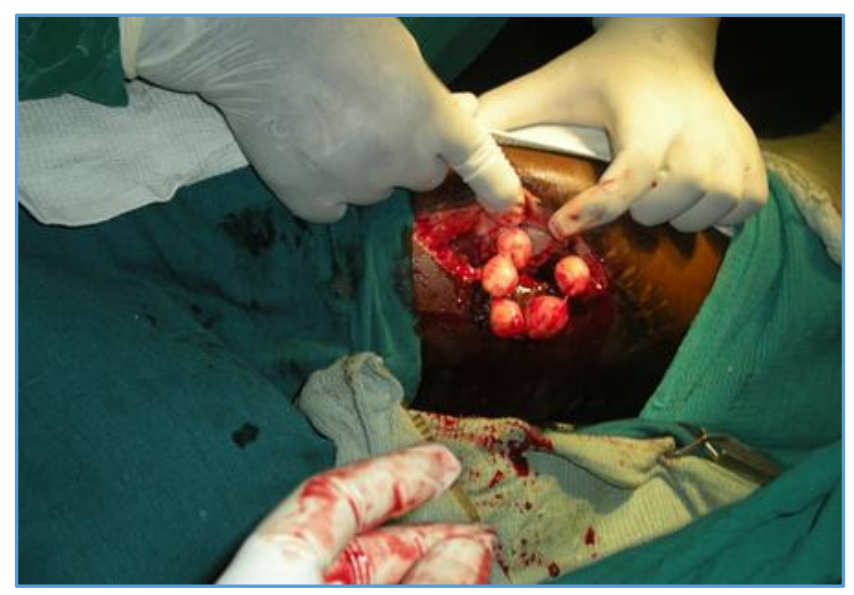

Figure 2. Application of Beads in Infected Region

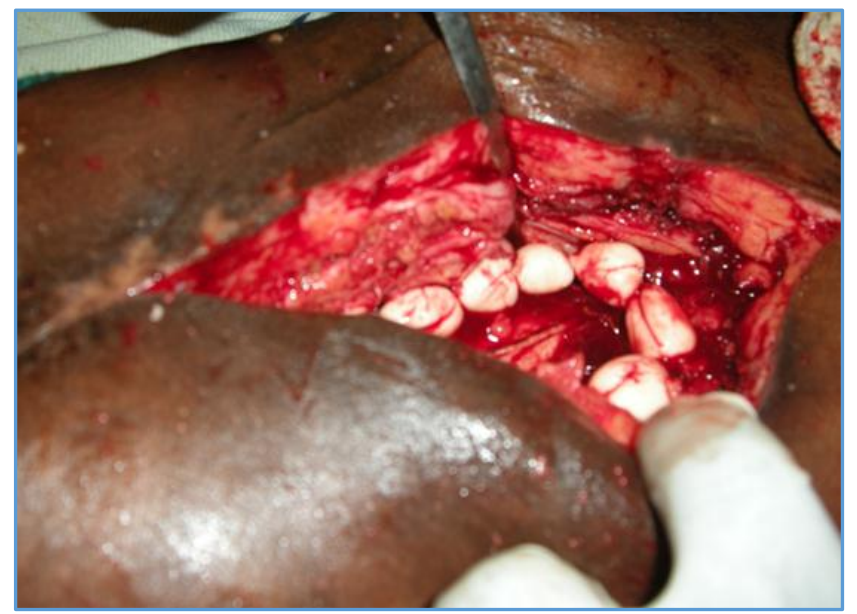

Figure 3. Securing of the Beads 


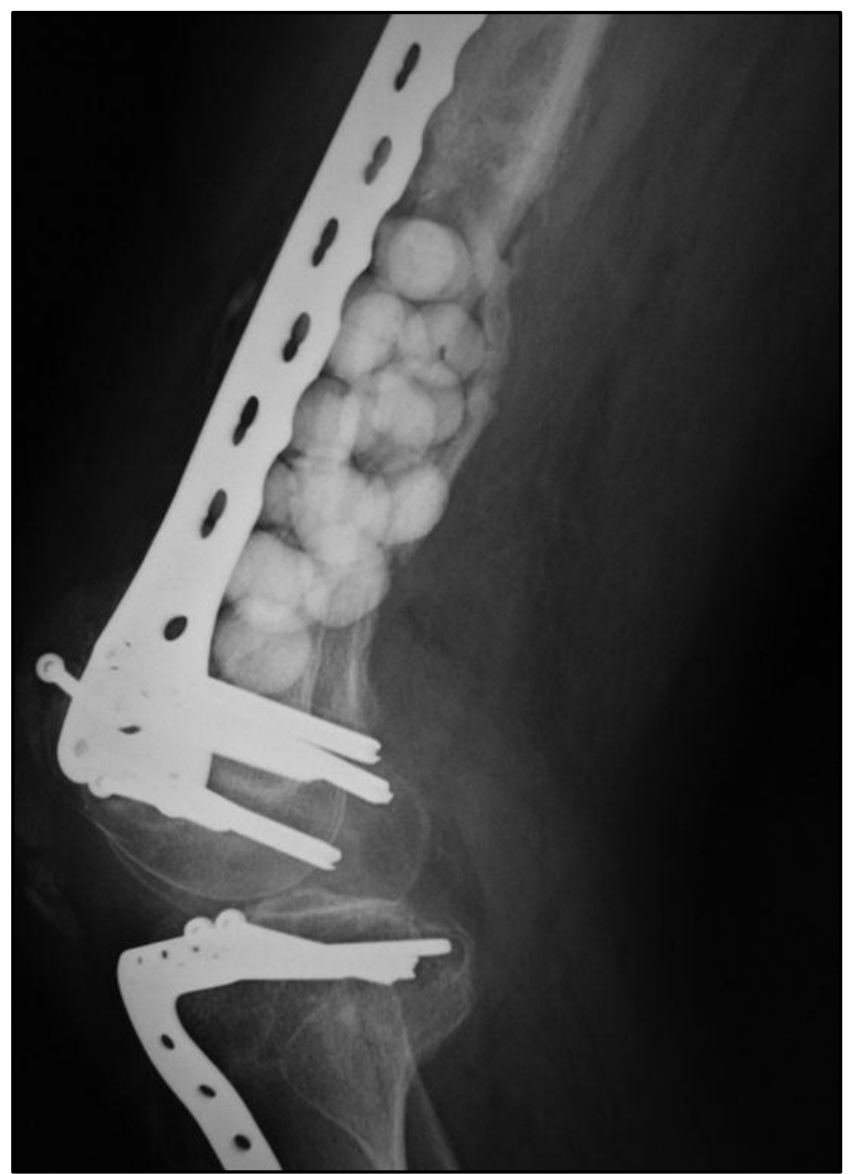

Figure 4. Antibiotic Beads in Infected Distal Femur Plate

\section{Drawback}

The main drawback of this procedure is that it needs a second procedure for bone cement removal after 4 to 6 weeks by which time, the antibiotic delivery reduces to almost nil and the risk of the cement harbouring the infection rises, so patient compliance for the second procedure is a must. The removed beads should be sent for culture and sensitivity.

\section{RESULTS}

We did this study in a series of 40 patients with infected implant in various bones. We chose those patients who had MRSA infection verified by culture and sensitivity so that our choice of antibiotic Vancomycin was in play. Postoperatively, we removed the beads in all of these patients by 6 weeks. Of these patients, 38 of them had appreciable results with a negative culture from wound swab after just one week and 36 of them remained infection free and proceeded till successful fracture union. Two of the patients had a recurrence of infection but as the fracture was united, we proceeded with implant exit, which still is a successful result. 6 patients had persistent infection and had to be treated with implant exit and alternate method of fixation viz. external fixation. The success rates were bone specific with femur fracture healing well than tibia and humerus fractures. In one of the patients with a tibia fracture with infected tibia intramedullary nail, we removed the infected nail and we chose a smaller diameter nail and covered it with the antibiotic cement and then did exchange nailing with the antibiotic coated nail. We removed the nail after successful union of the fracture.

We compared the results with patients treated with implant exit and found that both methods of treatment had a successful rate of control of infection and treatment with antibiotic beads had an almost similar success rate, thus proving to be an efficient alternative with further developments.

\section{DISCUSSION}

\section{Phenomenon of BIOFILM(7)}

Bacteria adhering to implant surfaces change their biological behaviour. They produce a biofilm that creates a protective microenvironment against antibiotics (Diefenbeck et al., 2006). More importantly, these micro-organisms reduce their metabolic activity and increase their generation time. As antibiotics act on growing bacteria, the minimum inhibitory concentrations (MIC) of micro-organisms with reduced metabolic activity is increased. Systemic antibiotics are ineffective as they cannot reach such a high concentration at the local site. Local antibiotic delivery is therefore a very viable option for this clinical problem, especially if retention of stable implants is planned. Some suggested, that the production of a kind of glycocalyx by the bacteria (Extracellular structure that covers the external surface of tissues with a "sheath" that is found mainly in epithelia), which adheres to the biomaterial, causes physiological changes on bacteria themselves and confers antibiotic resistance. Others proposed that the main factor can be the hydrophobicity of the implanted material, the electrostatic interactions and/or the roughness of the surface. Emerging evidences showed that the bacterial adhesion to a biomaterial is the result of a development of the antibiotic resistance.(2) It was hypothesised that the bacterial growth is privileged on certain biomaterials: For example, coagulase negative staphylococci would prefer to join the bone cement, while S. aureus would show preferential adhesion to metallic biomaterials. Prolonged exposure to antibiotic at a dose concentration below the inhibitory one, allows the development of mutational resistance in bacteria. Therefore, the wide clinical use of ALBC with preventive purposes must be carefully considered. The use of cement added with gentamicin for first implants was associated with the development of coagulase-negative staphylococci resistant to this drug.(6)

\section{Choice of Antibiotic}

The choice of antibiotics is determined by its thermostability property. Polymerisation process of cement is an exothermic reaction which releases substantial amount of heat. Hence only drugs that are thermostable can be used. Before an antibiotic is chosen to be used with the polymethylmethacrylate (PMMA) cement, many factors must be considered, including spectrum, heat stability, and elution characteristics. ${ }^{8,9}$ ) A synergistic effect has been seen with combinations of antibiotics [e.g. Vancomycin and Tobramycin used together.(10)] Vancomycin concentrations increased by $103 \%$ and Tobramycin by $68 \%$ when used together compared with their elution rates when used alone, showing passive opportunism.(11) This will, in essence, increase concentrations of antibiotics at the site locally, which will increase the bactericidal potential but also create a larger antimicrobial spectrum. The antibiotic also must be watersoluble, to permit diffusion into surrounding tissues while 
allowing a gradual release over time for a sustained bactericidal effect. The most commonly used antibiotics include Tobramycin, Gentamicin, Vancomycin, and Cephalosporins. Most periprosthetic infections involve Grampositive organisms (Staphylococcus aureus and Staphylococcus epidermidis), and when the pathogen is MRSA, vancomycin is a good choice.

One study conducted using Tobramycin as the antibiotic added to the Simplex cement showed good activity against $98 \%$ of the bacterial infections common in orthopaedic surgeries (aerobic Gram-positive and Gram-negative bacteria, anaerobic ones, and Mycobacterium).(12) With conventional antibiotic treatment, Tobramycin resistant bacteria such as Enterococcus faecalis, methicillin-resistant staphylococci, and Staphylococcus epidermidis fail to get controlled. But with Tobramycin elution locally from the cement, bacteria seemed to exhibit sensitivity to the antibiotic released, though sensitivity being limited. Thus, this study showed the effectiveness of Tobramycin coated cement for the prevention and reduction of infections caused by a wide spectrum of micro-organisms. Tobramycin being thermostable during the polymerisation process, which is exothermic in nature, starts eluting from the surface of PMMA later thereby slowly increasing concentration of Tobramycin that usually inhibits the growth of the majority of the bacteria.(13) Another study from the same authors compared the two principal aminoglycosides used in orthopaedic surgery, gentamicin and Tobramycin. Gentamicin was added to Palacos cement while Tobramycin was added to Simplex Cement. The results showed that Simplex-Tobramycin has better antibacterial activity than Palacos-Gentamicin combination against various strains of $\mathrm{P}$. aeruginosa. While the efficacy was $98 \%$ in Simplex-Tobramycin combination, it was $93 \%$ in Palacos-Gentamicin combination. Thus, it is proved that Tobramycin is better than Gentamicin in antibacterial activity. The efficacy of aminoglycosides increases with the concentration, therefore increasing the dose of the antibiotics available corresponds to an increased antibacterial efficacy. In addition, the elution of antibiotic is directly proportional to the quantity added to the cement.

Commercially available bone cements include Simplex P (Stryker Howmedica Osteonics, Mahwah, New Jersey), which contain $1 \mathrm{~g}$ of Tobramycin; SmartSet GHV and MHV (DePuy Orthopaedics, Warsaw, Indiana), which contain $1 \mathrm{~g}$ of gentamicin; and Palacos G (Biomet, Warsaw, Indiana), which contains $0.85 \mathrm{~g}$ of gentamicin.(11) These low-dose antibioticloaded cements are not effective in established infection. Therefore, surgeons need to add antibiotics to the cement in order to achieve the high doses in the infected region. In our study, we used $1 \mathrm{~g}$ of Vancomycin powder with $40 \mathrm{~g}$ of bone cement.

\section{Delivery of High Levels of Antibiotic from Bone Cement.(14)}

The elution of antibiotics from bone cement depends on several factors. The type of antibiotic, the amount and number of antibiotics, the porosity and type of cement, and the surface area of the spacer all play a role in the release.(15) Stevens et al studied the in vitro elution of antibiotics from Simplex and Palacos bone cements and found Palacos to be a more effective vehicle for local drug delivery. Elution of antibiotics from PMMA beads follows a biphasic pattern, with an initial rapid phase and a secondary slow phase.(16) In a study of the long term elution of antibiotics from polymethylmethacrylate bone cement in vivo in forty patients, Masri et al(17) found that effective levels of antibiotics remained four months after the operation. To maintain elution rates and concentrations sufficient to treat an established musculoskeletal infection, a minimum of $3.4 \mathrm{~g}$ of antibiotic should be hand mixed into each $40 \mathrm{~g}$ batch of PMMA and fashioned into beads or a cement spacer. The elution kinetics of antibiotic from acrylic cement follows a biphasic profile, with high elution rates early, followed by slower but sustained elution rates as time progresses.(14) The amount of antibiotic released from cement shows an exponential decline after day 1 of implantation. The hydrophobicity of ALBC permits roughly $10 \%$ of the added antibiotic to elute over a 6-8 week period. The mechanism by which antibiotic elutes from PMMA is not fully understood but generally is thought to be a passive phenomenon in which antibiotic diffuses out of pores, cracks, and voids in the cement.(13) The elution of antibiotic from PMMA is therefore dependent on multiple variables: which cement is used, which antibiotic is chosen, the amount of total antibiotic added, and how it is mixed. Highly porous cement has been shown to elute more antibiotic and for a longer period of time relative to cement with less porosity. Most studies evaluating combinations of antibiotics have demonstrated a synergistic effect, in that adding a second antibiotic seems to increase the elution of both antibiotics. This observation is consistent with the suggestion that at least $3.6 \mathrm{~g}$ of Tobramycin per $40 \mathrm{~g}$ of bone cement, with the addition of $1 \mathrm{~g}$ of Vancomycin, is an effective antibiotic regimen in this setting.(2)

The positioning into the surgical site of cement loaded with antibiotics may be useful to maintain at local level a high concentration of drug, which could not be reached by the venous administering without general complications and toxicity. After the surgical treatment and cleaning, the systemic administering of antibiotics for prolonged time is anyway mandatory.

\section{Toxicity of ALBC.(13)}

There are no reports in literature of systemic toxicity related to the use of ALBC. Various researches focused about local toxicity, with particular interest in the function of osteoblasts and osteocytes. Even though there are no reports of clinical adverse effects on these cells, some in vitro studies raised doubts about this subject. In addition, the concerns are more consistent in case of cement loaded at high doses, where the local levels of antibiotics may exceed $200 \mu \mathrm{g} / \mathrm{mL}$. When osteoblasts derived from trabecular bone were exposed to materials containing various concentrations of gentamicin $(0$ to $100 \mu \mathrm{g} / \mathrm{mL}$ ), the activity of alkaline phosphates decreased significantly in all the cultures with gentamicin concentration $>100 \mu \mathrm{g} / \mathrm{mL}$, the incorporation of $3 \mathrm{H}$-thymidine decreases at the same concentration of antibiotic, and the total DNA decreases for concentrations $\geq 700 \mu \mathrm{g} / \mathrm{mL}$. A study about the effect of Tobramycin (concentrations between 0 and 10,000 $\mu \mathrm{g} / \mathrm{mL}$ ) on osteoblasts showed that local levels $<200 \mu \mathrm{g} / \mathrm{mL}$ have no effect on replication of these cells, whereas at concentrations $>400 \mu \mathrm{g} / \mathrm{mL}$ replication decreases, and with $10,000 \mu \mathrm{g} / \mathrm{mL}$ cell death occurs.(13) Also the effects of Vancomycin on osteoblasts were studied for concentrations ranging between 0 and $10,000 \mu \mathrm{g} / \mathrm{mL}$ : levels of 
Vancomycin $<1.000 \mu \mathrm{g} / \mathrm{mL}$ had little or no effect on replication, but concentrations of $10,000 \mu \mathrm{g} / \mathrm{mL}$ caused the death of the osteoblasts. Vancomycin seems to be less toxic than aminoglycosides at high concentrations and Gentamicin has lower critical concentrations(18) than those of Tobramycin, despite they are both aminoglycosides.

\section{CONCLUSION}

Based on our experience, early aggressive debridement coupled with broad-spectrum antibiotic cement-coated beads are as effective as implant exit in control of infection and provides a better alternative. This approach permits fracture stabilisation and local delivery of high concentrations of broad-spectrum antibiotics and can reduce the number of debridement procedures required in the operating room.

Local high dose antibiotic delivery system using antibiotic impregnated bone cement was found to be highly effective in our study with $80 \%$ success rate and preventing further multiple procedures to the patient. It is clear how ALBCs are more effective than simple cements, but undoubtedly the "window of effectiveness" cannot be attributed only to antibiotics. Other properties related to the cement itself such as roughness, porosity, technique of preparation, and many patient-related features must be reminded.

However, it is necessary to underline that ALBC, especially if targeted by a specific antibiogram or integrated with an association of molecules more than a single one, is an important aid in the prevention and in the treatment of prosthetic infections.

So, this is a boon in management of such cases and in future better drug delivery systems can further improve the results in these patients.

\section{REFERENCES}

[1] Elson RA, Jephcott AE, McGechie DB, et al. Antibioticloaded acrylic cement. J Bone Joint Surg $\mathrm{Br}$ 1977;59(2):200-5.

[2] Minelli BE, Benini A, Biscaglia R, et al. Release of gentamicin and vancomycin alone and in combination from polymethylmethacrylate (PMMA) cement. Abstract to 21st International Congress of Chemotherapy, Birmingham, UK, July 4-7, 1999.

[3] Baker AS, Greenham LW. Release of gentamicin from acrylic bone cement. Elution and diffusion studies. J Bone Joint Surg Am 1988;70(10):1551-7.

[4] Garvin KL, Evans BG, Salvati EA, et al. Palacos gentamicin for the treatment of deep periprosthetic hip infections. Clin Orthop Relat Res 1994;298:97-105.
[5] Raut VV, Siney PD, Wroblewski BM. One-stage revision of total hip arthroplasty for deep infection. Long-term followup. Clin Orthop Relat Res 1995;321:202-7.

[6] Ivarsson I, Wahlström 0, Djerf $K$, et al. Revision of infected hip replacement. Two-stage procedure with a temporary gentamicin spacer. Acta Orthop Scand 1994;65(1):7-8.

[7] Minelli BE, Benini A, Magnan B, et al. Antibiotic release from removed temporary hip spacers. Abstract to 9th European Congress of Clinical Microbiology and Infectious Diseases, Berlin, Germany, March 21-24, 1999.

[8] Carlsson AS, Josefsson G, Lindberg L. Revision with gentamicin-impregnated cement for deep infections in total hip arthroplasties. J Bone Joint Surg Am 1978;60(8):1059-64.

[9] Marks KE, Nelson CL, Lautenschlager EP. Antibioticimpregnated acrylic bone cement. J Bone Joint Surg Am 1976;58(3):358-64.

[10] Penner MJ, Masri BA, Duncan CP. Elution characteristics of vancomycin and tobramycin combined in acrylic bone-cement. J Arthroplasty 1996;11(8):939-44.

[11] Duncan CP, Masri BA. The role of antibiotic-loaded cement in the treatment of an infection after a hip replacement. J Bone Joint Surg Am 1994;76(11):174251.

[12] Buchholz HW, Elson RA, Heinert K. Antibiotic-loaded acrylic cement: current concepts. Clin Orthop Relat Res 1984;190:96-108.

[13] Duncan CP, Beauchamp C. A temporary antibioticloaded joint replacement system for management of complex infections involving the hip. Orthop Clin North Am 1993;24(4):751-9.

[14] Callaghan JJ, Katz RP, Johnston RC. One-stage revision surgery of the infected hip. A minimum 10-year followup study. Clin Orthop Relat Res 1999;369:139-43.

[15] Salvati EA, Callaghan JJ, Brause BD, et al. Reimplantation in infection. Elution of gentamicin from cement and beads. Clin Orthop 1986;207:83-93.

[16] Kuechle DK, Landon GC, Musher DM, et al. Elution of vancomycin, daptomycin and amikacin from acrylic bone cement. Clin Orthop Relat Res 1991;264:302-8.

[17] Coventry MB. Treatment of infections occurring in total hip surgery. Orthop Clin North Am 1975;6(4):991-1003.

[18] Wahlig H, Dingeldein E, Buchholz HW, et al. Pharmacokinetic study of Gentamicin-loaded cement in total hip replacements. Comparative effects of varying dosage. J Bone Joint Surg Br 1984;66(2):175-9. 